\title{
A Geant4 simulation code for simulating optical photons in SPECT scintillation detectors
}

\author{
S. Lo Meo, ${ }^{a, b, 1}$ P. Bennati, ${ }^{c, e}$ M.N. Cinti, ${ }^{c, d}$ N. Lanconelli, ${ }^{a, b}$ F.L. Navarria, ${ }^{a, b}$ R. Pani, ${ }^{c, d}$ \\ R. Pellegrini, ${ }^{c, d}$ A. Perrotta ${ }^{a}$ and F. Vittorini ${ }^{d}$ \\ ${ }^{a}$ INFN Bologna, \\ Viale Berti Pichat 6/2, 40127 Bologna, Italy \\ ${ }^{b}$ Dept. of Physics, Alma Mater Studiorum - University of Bologna, \\ Viale Berti Pichat 6/2, 40127 Bologna, Italy \\ ${ }^{c}$ INFN Rome, \\ P.le Aldo Moro 5, 00185 Rome, Italy \\ ${ }^{d}$ Dept. of Experimental Medicine "Sapienza" University of Rome, \\ Viale Regina Elena 272, 00161 Rome, Italy \\ ${ }^{e}$ EDEMOM Ph.D. School on Microelectronics, University of Rome III, \\ Via della Vasca Navale 84, 00146 Rome, Italy \\ E-mail: sergio.lomeo@bo.infn.it
}

ABSTRACT: Geant4 is an object oriented toolkit created for the simulation of High-Energy Physics detectors. Geant 4 allows an accurate modeling of radiation sources and detector devices, with easy configuration and friendly interface and at the same time with great accuracy in the simulation of physical processes. While most Monte Carlo codes do not allow the simulation of the transport and boundary characteristics for optical photons transport generated by scintillating crystal, Geant 4 allows the simulation of the optical photons. In this paper we present an application of the Geant 4 program for simulating optical photons in SPECT cameras. We aim to study the light transport within scintillators, photomultiplier tubes and coupling devices. To this end, we simulated a detector based on a scintillator, coupled to a photomultiplier tube through a glass window. We compared simulated results with experimental data and theoretical models, in order to verify the good matching with our simulations. We simulated a pencil beam of $140 \mathrm{keV}$ photons impinging the crystal at different locations. For each condition, we calculated the value of the Pulse Height Centroid and

${ }^{1}$ Corresponding author. 
the spread of the charge distribution, as read out by the anode array of the photomultiplier. Finally, the spatial and the energy resolutions of the camera have been estimated by simulated data. In all cases, we found that simulations agree very well with experimental data.

KEYWORDS: Scintillators, scintillation and light emission processes (solid, gas and liquid scintillators); Gamma camera, SPECT, PET PET/CT, coronary CT angiography (CTA); Detector modelling and simulations I (interaction of radiation with matter, interaction of photons with matter, interaction of hadrons with matter, etc) 


\section{Contents}

1 Introduction $\quad 1$

2 Materials and methods $\quad 2$

3 Results 3

\section{Introduction}

Monte Carlo simulation techniques are becoming very common in the medical imaging community. Several topics were addressed by Monte Carlo simulations in the Nuclear Medicine field. Among them, it is worth remarking the optimization of imaging system design (including detector, collimator, and shield design), the development of correction methods for improved image quantitation, the evaluation of correction techniques (scatter, random, attenuation corrections, partial volume effect), the assessment of image reconstruction algorithms, ROC studies, pharmaco-kinetic modeling.

In Nuclear Medicine the use of Monte Carlo methods was profited from the possibility of using general purpose codes developed for high energy physics. Various general purpose Monte Carlo codes, initially developed to simulate particle transport in a broad context (such as EGS and Geant), can be used also for modeling SPECT and PET configurations. As they have been designed for a large community of researchers, these codes are well documented and in the public domain. Our group has already used and validated various Monte Carlo codes for simulating imaging systems with applications in Nuclear Medicine and industry [1-3].

Geant4 [4] is an object oriented toolkit for simulation of current (and next generation) HighEnergy Physics detectors. It is also a showcase example of technology transfer from particle physics to other fields such as medical science [5]. Geant4 allows an accurate modeling of radiation sources and detector devices with easy configuration and friendly interface and at the same time with great accuracy on the description of physical processes. In the last releases the user is able to simulate electromagnetic interactions at energies as small as $1 \mathrm{keV}$. While most Monte Carlo codes do not allow the simulation of the transport and boundary characteristics for optical photons generated by scintillating crystal, in Geant 4 two optical models can be used for this purpose: either GLISUR or UNIFIED model [6]. The purpose of this paper is to present an application of Geant4 program for simulating the optical photons transport in the different components of SPECT cameras. We aim to study the light transport within scintillators, photomultiplier (PM) tubes and coupling devices. To this end, we simulated a detector based on a scintillator, coupled to a PM through a glass window. Simulations were also compared with experimental results obtained in the same configuration. 


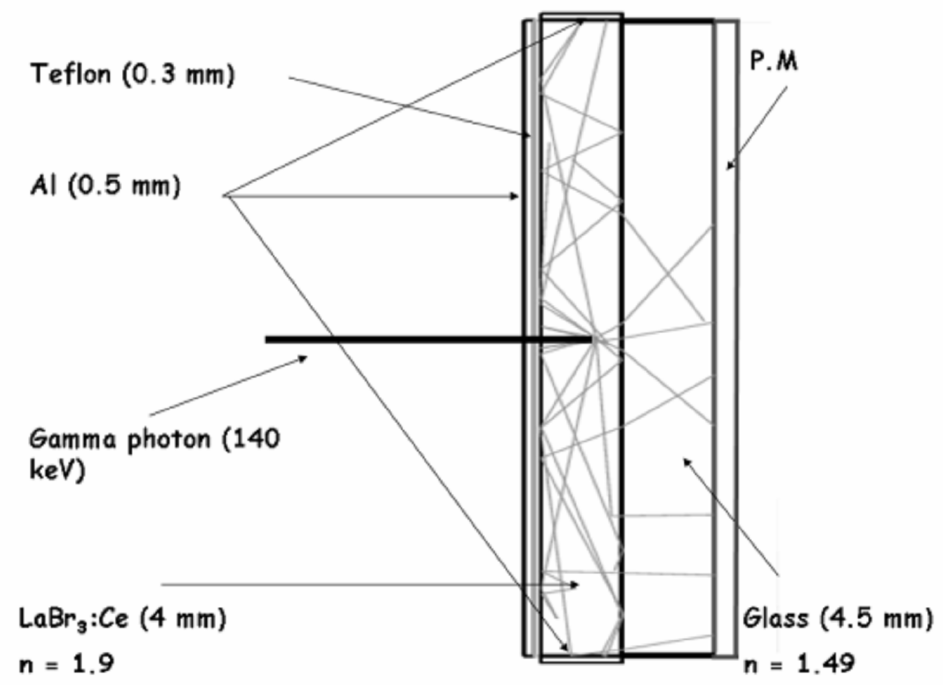

Figure 1. Example of the tracking of the optical photons simulated within the detector with Geant4. The detector is simulated as composed of three components: a crystal, a glass window for the optical coupling of the crystal with the photomultiplier, and the photomultiplier itself. The front surface of the crystal is composed of an Aluminum layer and a Teflon layer.

\section{Materials and methods}

The experimental setup is based on the $5 \times 5 \mathrm{~cm}^{2}$ scintillation gamma camera described in [7]. The detector consists on a multi anode PMT Hamamatsu Flat Panel H8500 [8], coupled to a $50 \times 50 \times$ $4 \mathrm{~mm}^{3}$ continuous $\mathrm{LaBr}_{3}: \mathrm{Ce}$ crystal [9] and a $4.5 \mathrm{~mm}$ glass window. The crystal is surrounded by a thin layer of Aluminum. In the front crystal surface a very small layer of Teflon is positioned after the Aluminum. A $0.4 \mathrm{~mm}$ collimated ${ }^{99 \mathrm{~m}} \mathrm{Tc}$ spot source was used to analyze the spread of the charge distribution on the anodic array and the intrinsic spatial resolution of the detector.

With Geant 4 we simulated a system resembling the above described experimental apparatus. The scintillation light photons are generated as a pure Poisson process. The simulated crystal was wrapped in a material acting as a Lambertian reflector (Teflon on front) or Black absorber (Aluminum). The interface was modeled as ground, and the boundary processes followed the rules of the GLISUR model. In this model the user can select the smoothness or the roughness of each surface surrounding the crystal by using a parameter named polish. For example, polish equal to zero corresponds to the maximum roughness (photons are reflected according to a Lambertian distribution), whereas for polish equal to one the Snell's Law is applied. For each surface one can also select the value of reflectivity. The PM surface is simulated as a polished quartz window, assuming an experimentally derived value for quantum efficiency. The simulated setup also consists of monochromatic point sources ( $140 \mathrm{keV}$ photons). The Geant 4 application was run to record the spatial distribution of the detected optical light photons on the PM surface and to estimate the energy resolution of the crystal (figure 1). 


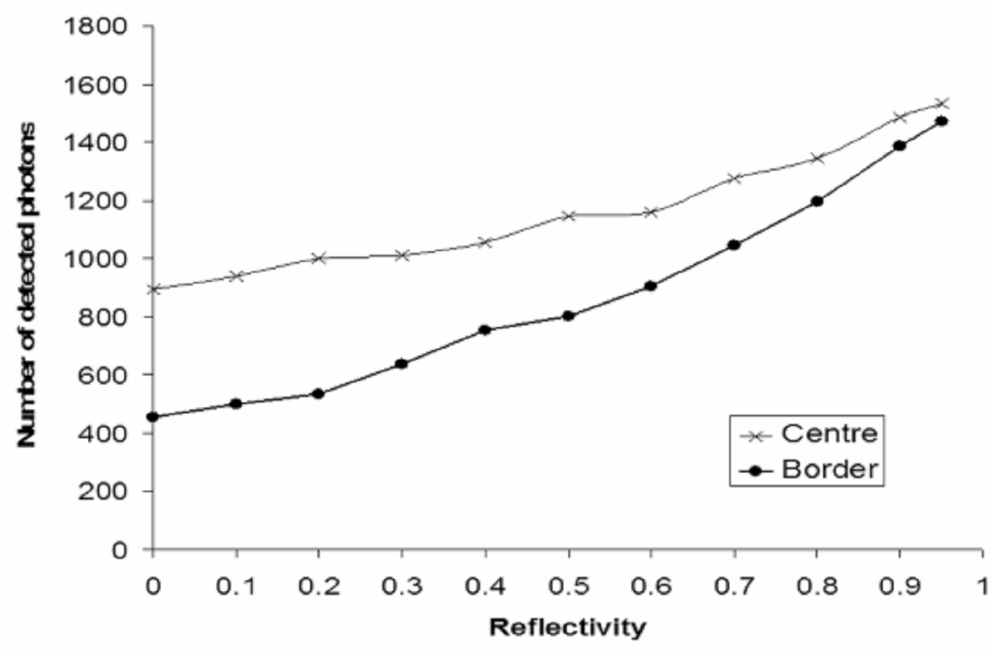

Figure 2. Number of detected photons with the gamma impinging at the centre and at the border of the crystal, as a function of the reflectivity of the crystal's lateral surfaces.

\section{Results}

We compared Monte Carlo results with experimental data and theoretical models, in order to verify the good matching with our simulations. First of all, with Geant 4 we simulated a pencil beam of $140 \mathrm{keV}$ photons interacting with the $\mathrm{LaBr}_{3}$ :Ce crystal. Figure 1 shows an example of the tracking of the optical photons inside the detection camera. Here, one $140 \mathrm{keV}$ photon impinging the scintillator $\left(\mathrm{LaBr}_{3}: \mathrm{Ce}\right)$ from the left generates some optical photons. Some of them travel directly through the glass window towards the photomultiplier. Some others are reflected by the lateral surfaces or by the front side of the crystal. The number of optical photons generated within the scintillation crystal was fixed at $63000 / \mathrm{MeV}$ and the mean energy of these optical photons was $3 \mathrm{eV}(380 \mathrm{~nm})$ [9]. The fraction of optical photons moving towards the glass resulted to be $44.9 \%$ in the simulation, while the theoretical expectation, based on purely geometric arguments, is about $45 \%$. The fraction of optical photons moving towards the PM was $17.9 \%$, to be compared with an expectation of about $19 \%$. We assumed, as coming from experimental data, that the quantum efficiency of the PM was 0.22 .

We have then estimated the number of optical photons detected by the PM, both when the gamma photon interacts at the centre and close to the border of the crystal. We calculated the number of detected optical photons in correspondence of different values of the reflectivity of the crystal outer surface. Experimental data show that the number of photons reaching the PM with the gamma at the border is about $25 \%$ less than the number of photons reaching the PM with gamma interacting at the centre. Figure 2 shows the number of detected photons with the gamma impinging at the centre and at the border of the crystal, as a function of the reflectivity of the crystal's lateral surfaces.

We found that the best agreement between experimental and simulated data is gained with polish equal to zero, $\mathrm{R}=0.6$ for the crystal's lateral surfaces (top, bottom, right and left), and $\mathrm{R}=0.95$ for the front surface. On the other hand, we applied the Snell's Law for the surface between crystal and PM glass (parameter polish fixed to 1). 


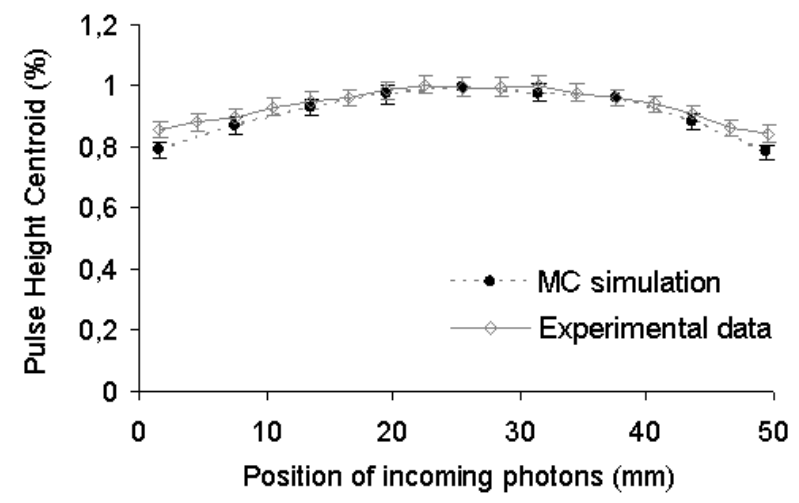

Figure 3. Pulse Height Centroid values for an impinging photon of $140 \mathrm{keV}$ scanning the crystal.

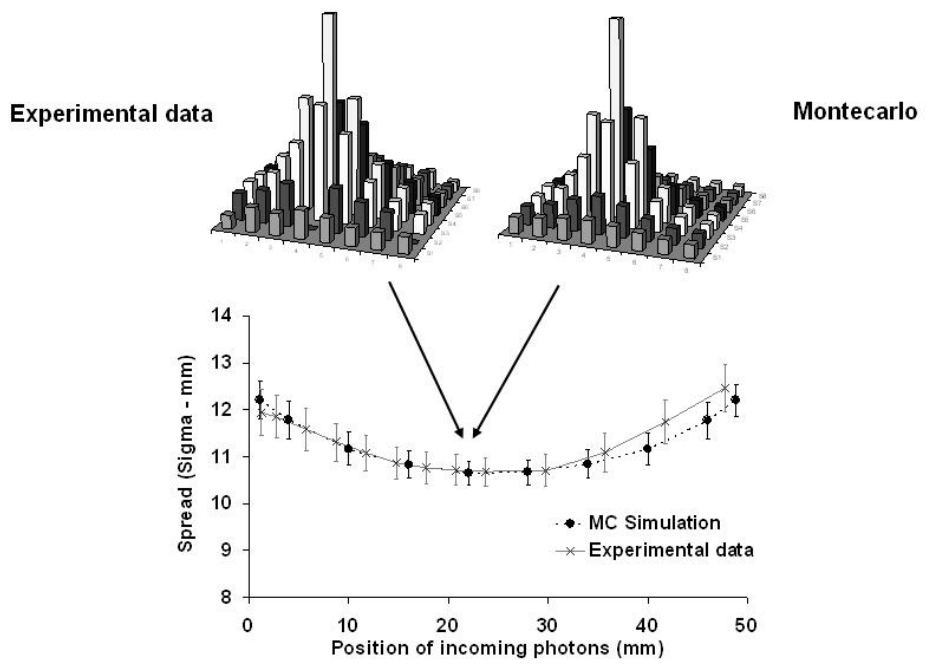

Figure 4. Charge distribution spread into the PM anode array for an impinging photon of $140 \mathrm{keV}$ scanning the crystal.

We simulated the output of the PM as a $8 \times 8$ pixellated anode, where every pixel has a front surface of $6 \mathrm{~mm} \times 6 \mathrm{~mm}$, as in the experimental setup. We scanned the crystal, with a pencil beam of $140 \mathrm{keV}$ energy photons, from one border to the other, passing through the centre. For each position of the impinging photon we estimated the Pulse Height Centroid, as acquired in the PM anode array. Figure 3 and 4 show the Pulse Height Centroid values and the spread of the charge distribution into the PM anodes for the different positions of the incoming photons, respectively. It is worth noting that there is an excellent agreement between the simulations and the experimental data.

Finally, we compared the spatial and the energy resolutions obtained with Geant 4 with experimental data, by using a pencil beam of $140 \mathrm{keV}$ energy photons that interacts in the centre of the crystal. With the simulation we obtained a spatial resolution of $0.9 \mathrm{~mm}$ (FWHM) and an energy resolution of $6.7 \%$ (FWHM), while the experimental data provide $1.1 \mathrm{~mm}$ and $6.9 \%$, respectively. Again, simulations agree very well with experimental data. 
In summary, we investigated some characteristics concerning the transport of light photons within a SPECT camera. We simulated the optical features of the various components of the camera (scintillator, glass coupling window, and photomultiplier), and compared results with the same experimental configuration. Simulated outcomes, in terms of Pulse Height Centroid values, charge spread distribution, spatial and energy resolutions agree very well with experimental data. The results of this work will be used in the near future to arrange the components of the camera and optimize its performance

\section{References}

[1] S. Vecchio et al., A single photon emission computer tomograph for breast cancer imaging, Nucl. Instrum. Meth. 581 (2007) 84.

[2] A. Berdondini et al., Monte Carlo optimization of an industrial tomography system, Nucl. Instrum. Meth. A 580 (2007) 771.

[3] M.N. Cinti et al., CsI(Tl) Micro-Pixel Scintillation Array for Ultra-high Resolution Gamma-ray Imaging, IEEE Trans. Nucl. Sci. 54 (2007) 469.

[4] S. Agostinelli et al., GEANT4 - a simulation toolkit, Nucl. Instrum. Meth. A 506 (2003) 250.

[5] J. Allison et al., Geant4 developments and applications, IEEE Trans. Nucl. Sci. 53 (2006) 270.

[6] A. Levin and C. Moisan, A more physical approach to model the surface treatment of scintillation counters and its implementation into DETECT, IEEE Nucl. Sci. Symp. Conf. Rec. 2 (1996) 702.

[7] R. Pani et al., LaBr 3 :Ce scintillation gamma camera prototype for $X$ and gamma ray imaging, Nucl. Instrum. Meth. A 576 (2007) 15.

[8] Hamamatsu Photonics, H8500 PSPMT data sheet, Japan, available at:www.hamamatsu.com.

[9] Saint Gobain 2006, BrillianceTM380 Technical data sheet, available at: www.detectors.saint-gobain.com. 